\title{
Histology at transurethral resection of bladder tumor and radical cystectomy for bladder cancer: Insights from population-based data
}

\author{
Tina Dyer ${ }^{1,2}$; D. Robert Siemens ${ }^{3,4}$; Pria Nippak ${ }^{2}$; Julien Meyer²; Christopher M. Booth ${ }^{1,4}$ \\ ${ }^{1}$ Division of Cancer Care and Epidemiology, Queen's Cancer Research Institute, \\ Kingston, ON, Canada; ${ }^{2}$ School of Health Services Management, Ryerson University, Toronto, ON, Canada; \\ ${ }^{3}$ Department of Urology, Queen's University, Kingston, ON, Canada; ${ }^{4}$ Department of Oncology, Queen's \\ University, Kingston, ON, Canada
}

\begin{abstract}
Acknowledgments: The authors gratefully acknowledge the contributions of Weidong Kong in the conduct of this study. Parts of this material are based on data and information compiled and provided by Ontario Health-Cancer Care Ontario. However, the analyses, conclusions, opinions, and statements expressed herein are those of the authors, and not necessarily those of Ontario Health-Cancer Care Ontario. Dr. Booth is supported as the Canada Research Chair in Population Cancer Care.
\end{abstract}

Cite as: Dyer T, Siemens DR, Nippak P, et al. Histology at transurethral resection of bladder tumor and radical cystectomy for bladder cancer: Insights from population-based data. Can Urol Assoc J 2020 September 28; Epub ahead of print. http://dx.doi.org/10.5489/cuaj.6856

Published online September 28, 2020

$* * *$

\section{Introduction}

Transurethral resection of bladder tumour (TURBT) is the definitive diagnostic procedure for bladder cancer. Pathological findings including extent of disease (T stage), grade, and histology dictate subsequent steps in management. Pathological review at TURBT is, therefore, crucial to guide management. ${ }^{1}$

Like any diagnostic biopsy, TURBT provides a limited pathological sample to characterize the extent and biological risk of bladder cancer. There is little published data about quality of reporting and concordance between TURBT and radical cystectomy (RC) in routine clinical practice. In this study, we compare and contrast pathological findings at TURBT with subsequent findings at RC among all patients treated in Ontario from 2009-2013.

\section{Methods}

This retrospective population-based study reports timing and pathologic concordance of TURBT and RC among all patients treated with RC in Ontario during 2009-2013. This is a sub-study of a population-based, retrospective cohort study to describe management and outcome of urothelial carcinoma of the urinary bladder in the Canadian province of Ontario. Primary study results and 
methodology are reported elsewhere. ${ }^{2-3}$ All incident cases of bladder cancer in Ontario in patients who underwent cystectomy in 2009-2013 were identified using the Ontario Cancer Registry (OCR) and linked to treatment records. Stage of disease was not routinely available in the existing data sources; for this reason we obtained surgical pathology reports for all cystectomy cases. The study was approved by the Research Ethics Board of Queen's University.

The OCR is a passive, population-based cancer registry that captures diagnostic and demographic information on at least $98 \%$ of all incident cases of cancer diagnosed in the province of Ontario (approximate population 13,500,000). ${ }^{4-5}$ Records from the Canadian Institute for Health Information were used to identify those patients treated with RC. Surgical pathology reports were obtained from the OCR. A team of trained data abstractors reviewed the pathology reports and entered pathologic variables into an electronic database, capturing stage as assigned by the pathologist. To be eligible for the current study patients with $\mathrm{RC}$ were required to have a preceding TURBT pathology report available for review.

\section{Results}

\section{Study population}

From our database we identified pathology reports for 1647 patients treated with RC. Among these cases, we were able to identify a preceding TURBT pathology report for 1580 (96\%); this represents the study population. The characteristics of the study population are shown in Table 1. Two thirds $(1039 / 1580,66 \%)$ of patients had muscle-invasive disease at TURBT immediately preceding RC.

At time of TURBT, 541 (34\%) of patients had no evidence of invasion of muscularis propria and presumably received $\mathrm{RC}$ for high risk pathological or clinical features. Among these 541 cases, at time of RC, 303 (56\%) were upstaged to T2+ disease. Among 132 patients with CIS only at TURBT immediately prior to RC, 90 (68\%) were found to have invasive carcinoma at RC. Similarly, 244 of 369 (66\%) of cT1 cases were upstaged to >pT2 disease. Conversely, among the 1039 cases with T2+ disease at TURBT, $106(10 \%)$ were down staged to $<$ T2 at time of RC. The down staging at the time of RC may be attributed to the TURBT or utilization of NACT. We have previously reported that $19 \%$ of patients with MIBC in Ontario treated with RC during this time period received neoadjuvant chemotherapy. ${ }^{6}$

Histology at time of TURBT and RC indicate that 94\% of the pathology reports at TURBT prior to RC in Ontario describe a urothelial cancer although 231 (16\%) of these with concomitant squamous differentiation. The overall rate of variant or divergent pathology beyond squamous differentiation abstracted for the TURBT pathology reports was $6 \%$ and that for the RC reports was $6.8 \%$. Patients with variant histology at TURBT were commonly re-classified at $\mathrm{RC} ; 30 \%$ of patients with pure squamous cell histology and $25 \%$ with adenocarcinoma were reclassified as UC at time of RC. 


\section{Discussion}

In this study we explore histopathologic findings at time of TURBT and subsequent cystectomy among 1580 patients treated in Ontario during 2009-2013. Several important findings have emerged. First, we find that in routine clinical practice, one third of patients undergoing cystectomy had high-risk non-muscle invasive disease and two thirds had muscle-invasive disease at time or TURBT. Second, we observe substantial upstaging at time of RC; $56 \%$ of patients with $<\mathrm{T} 2$ disease at TURBT are found to have $\mathrm{T} 2+$ disease at time of RC. Third, the rate of down-staging $\mathrm{T} 2+$ at TURBT to $<\mathrm{T} 2$ disease at $\mathrm{RC}(10 \%)$ was lower than expected. Finally, we find that variant histology at TURBT is often re-classified as UC at time of RC in routine care.

A discrepancy between the initial clinical T-stage at TURBT and the final pathological stage is common, with both frequent under-staging (40-49\%) and over-staging (22-27\%). ${ }^{7-10}$ These discrepancies can have consequences for management and may be secondary to various factors including incomplete TURBT, limitations of conventional imaging as well as prolonged wait times between TURBT and RC. In the present study the accuracy of the upstaging findings is limited without knowledge of other clinical parameters such as imaging. However, the apparent down-staging of only $10 \%$ is remarkable. In a similar population-based study from Europe, excluding patients that received neoadjuvant therapies, the down-staging rates were approximately $26 \%{ }^{7}$ Although an explanation for this disparity in Ontario is not answerable from this study, one could hypothesize differences in bladder cancer management may play a role: aggressivity of TURBT, delayed diagnosis leading to higher volume disease, prolonged wait times.

The large variation in the reported incidence of divergent differentiation at TURBT (between 7-81\%) is likely due to differences in sampling and enhanced recognition of its import in more contemporary series. ${ }^{11}$ The reporting of variant pathology beyond squamous differentiation in routine care in Ontario appeared to be less common than previous studies suggesting inconsistent recognition or reporting. Interestingly, when we further look at the discrepancy between TURBT and RC for academic versus community hospitals, we observed that the rates of variant pathology for TURBTs indicated academic (10\%) vs community (5\%), $\mathrm{p}=0.0001$; and the rates of variant pathology for RC were academic (11\%) vs community $(5 \%)$, $\mathrm{p}<0.0001$.

Our results should be considered in light of methodologic limitations. This is a retrospective study and the pathological reports were not reviewed by central pathologists. Thus, we are evaluating the histopathologic reporting without confirming quality assurance. Although the pathology database used in this study describes general aspects of disease for patients in the province, the data source detailing information related to neoadjuvant and adjuvant chemotherapy, radiation and immunotherapy treatment was not linked to the pathology data collected and limits our ability to examine and evaluate survival outcomes. Furthermore, not 
having access to NMIBC intravesical therapy data, we were unable to determine whether or not there was upstaging among those patients who received intravesical BCG compared to those who did not for patients who underwent $\mathrm{RC}$ for $<\mathrm{cT} 2$ disease. However, because this was a population-based study aimed at elucidating practice patterns and outcomes in "the real world" with all these inherent limitations, it still offers important insight into care and outcomes in routine practice.

\section{Conclusions}

These data illustrate that in a substantial proportion of patients, the extent of disease and dominant histology at time to TURBT will be re-classified at RC. We also observe that the rate of down-staging observed in routine practice is lower than expected. The extent to which these findings reflect a differential treatment effect or inconsistent reporting is not clear. The results of our study lend further to the premise that TURBT and RC specimens be reviewed by a pathologist with expertise in urologic cancer and further lends to the premise that synoptic reporting may play a role in the assessment and a more accurate reporting of variant pathology. Further work is necessary to better understand these apparent inconsistencies in an effort to improve the quality of care delivered to patients with bladder cancer in the general population.

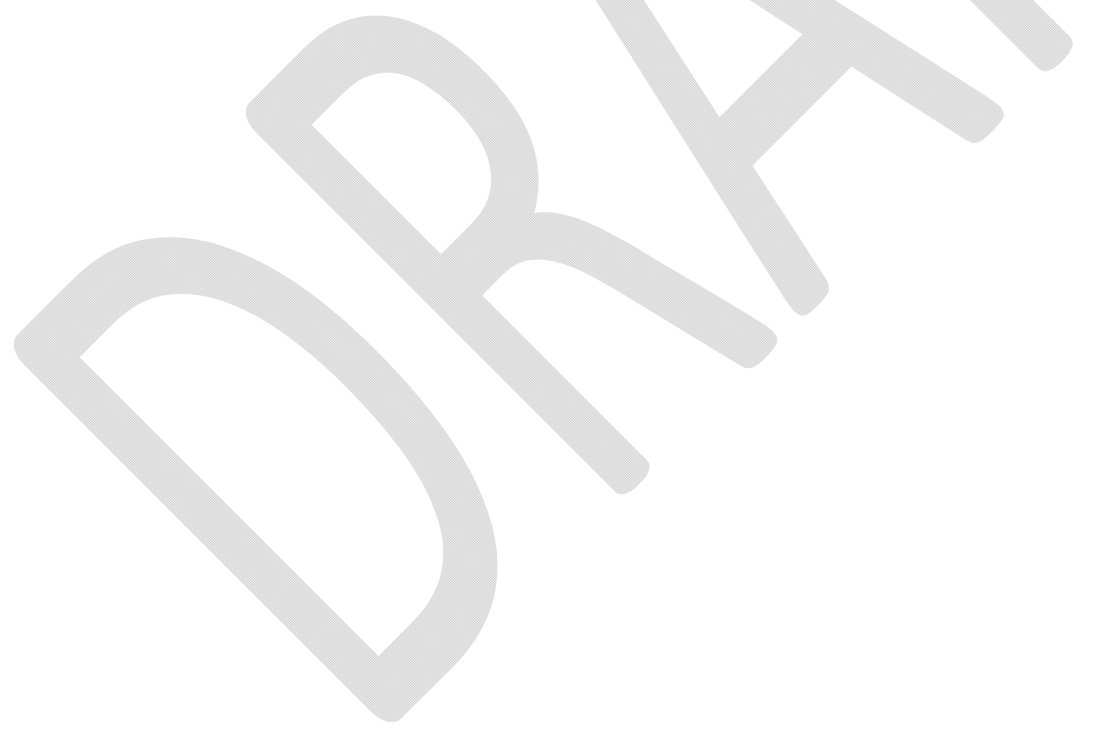




\section{References}

1. Kulkarni, G.S., Black, P.C., Sridhar, S.S., et al. Canadian Urological Association guideline: Muscle-invasive bladder cancer. Can Urol Assoc J 2019; 13:230-38. http://dx.doi.org/10.5489/cuaj.5902

2. Booth, C.M., Siemens, D.R., Li, G., et al. Curative Therapy for Bladder Cancer in Routine Clinical Practice: A Population-based Outcomes Study. Clinical Oncology 2014; 26:506-14. doi: https://doi.org/10.1016/j.clon.2014.05.007.

3. Booth, C. M., Siemens, Karim, S., et al. Perioperative chemotherapy for bladder cancer in the general population: Are practice patterns finally changing? Urol Oncol 2018; 36:89 e13-20. doi: 10.1016/j.urolonc.2017.11.015.

4. Health Quality Ontario. Measuring wait times for cancer surgeries. 2018; Retrieved from https://www.hqontario.ca/System-Performance/Measuring-SystemPerformance/Measuring-Wait-Times-for-Cancer-Surgeries

5. Cancer Care Ontario. Cancer treatments: Surgery. 2018; Retrieved from https://www.cancercareontario.ca/en/cancer-treatments/surgery

6. Booth, C.M., Siemens D. R., Li, G., et al. Perioperative chemotherapy for muscleinvasive bladder cancer. Cancer 2014; 120:1630-38. doi 10.1002/cncr.28510

7. van Dijk PR, Ploeg M, Aben KK, et al. Downstaging of TURBT-Based Muscle-Invasive Bladder Cancer by Radical Cystectomy Predicts Better Survival. ISRN Urol 2011; 2011: 458930. doi:10.5402/2011/458930. Epub 2011 Apr 27.

8. Shariat SF, Palapattu GS, Karakiewicz PI, et al. Discrepancy between clinical and pathologic stage: impact on prognosis after radical cystectomy. European Urology 2007; 51:137-51.

9. Hollenbeck BK, Miller DC, Dunn RL, et al. The effects of stage divergence on survival after radical cystectomy for urothelial cancer. Urologic Oncology: Seminars and Original Investigations 2005; 23:77-81. doi: 10.1016/j.urolonc.2004.08.012.

10. Pagano F, Bassi P, Galetti TP, et al. Results of contemporary radical cystectomy for invasive bladder cancer: a clinicopathological study with an emphasis on the inadequacy of the tumor, nodes and metastases classification. Journal of Urology 1991; 145:45-50. doi: 10.1016/s0022-5347(17)38244-7.

11. Chalasani V, Chin JL, Izawa JI. Histologic variants of urothelial bladder cancer and nonurothelial histology in bladder cancer. Can Urol Assoc J 2009; 3:193-98. doi:10.5489/cuaj.1195. 
Figures and Tables

\begin{tabular}{|c|c|}
\hline & n $(\%)^{* * *}$ \\
\hline \multicolumn{2}{|l|}{ Age (years) } \\
\hline$<50$ & $52(3)$ \\
\hline $50-60$ & $252(16)$ \\
\hline $61-70$ & $480(30)$ \\
\hline $70+$ & $796(50)$ \\
\hline \multicolumn{2}{|l|}{ Gender } \\
\hline Male & $1209(77)$ \\
\hline Female & $371(24)$ \\
\hline \multicolumn{2}{|l|}{ Histology } \\
\hline Urothelial & $1485(94)$ \\
\hline Squamous cell & $40(3)$ \\
\hline Adenocarcinoma & $23(1)$ \\
\hline Other histology ${ }^{*}$ & $32(2)$ \\
\hline \multicolumn{2}{|l|}{ LVI } \\
\hline Yes & $631(40)$ \\
\hline No & $768(49)$ \\
\hline Unstated & $181(12)$ \\
\hline \multicolumn{2}{|l|}{ Stage at TURBT } \\
\hline $\mathrm{TX}$ & $8(<1)$ \\
\hline $\mathrm{Ta}$ & $92(6)$ \\
\hline+2 & $72(5)$ \\
\hline $\mathrm{T} 1$ & $369(23)$ \\
\hline $\mathrm{T} 2+^{* * * *}$ & $1039(66)$ \\
\hline
\end{tabular}

* Other includes sarcomatoid transitional cell carcinoma, carcinoma not otherwise specified (NOS), squamous cell carcinoma sarcomatoid, small cell carcinoma NOS, signet ring adenocarcinoma. ${ }^{* *} \%$ may not add to $100 \%$ due to rounding. ${ }^{* * *} \mathrm{~T} 2+$ denotes $\mathrm{T} 2, \mathrm{~T} 3$, and $\mathrm{T} 4$. LVI: lymphovascular invasion; TURBT: transurethral resection of bladder tumor. 